\title{
A Chiral Magnet Induces Vortex Currents in Superconductors
}

\author{
Control over vortices that arise in magnet-superconductor
}

heterostructures could lead to qubits that are immune to the effects of

their environment.

By Gustav Bihlmayer

( uantum computers can solve difficult numerical problems significantly faster than conventional computers, but their realization is made trickier by errors resulting from the interaction of the quantum system with its environment. One promising approach for developing fault-tolerant quantum computers-ones that are resistant to errors due to interactions with the environment-is the concept of topological quantum computing. Here, operations are carried out on anyons-quasiparticles whose collective states are mostly immune to environmental effects. However,

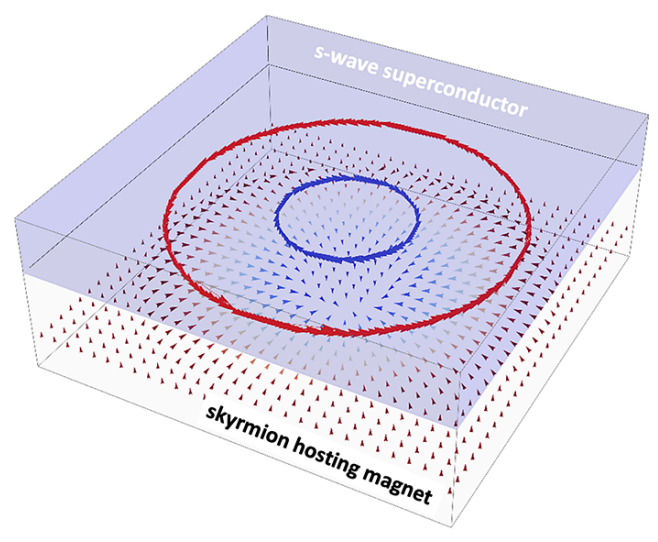

Figure 1: A heterostructure comprising a magnetic material (white) that hosts a type of skyrmion, coupled to a superconductor (blue) could provide a platform for hosting and manipulating quasiparticles that may be useful in quantum computing. The stray magnetic field from the skyrmion induces vortices in the superconducting layer. These skyrmion-vortex pairs may host stable hybrid topological excitations.

Credit: APS/Gustav Bihlmayer generating these quasiparticles in materials has proven challenging. Now, Alexander Petrović of Nanyang Technical University, Singapore, and colleagues have fabricated a 2D structure that models suggest could host anyons [1].

Anyons occur in two dimensions and are different than other particles, such as electrons and photons. In particular, anyons can obey non-Abelian statistics, which means that if you take some anyons and swap their positions, the final state of the system will depend on the sequence of the individual exchanges [2]. This path dependence could be used to encode information in a form that would be robust against localized perturbations, which is why non-Abelian anyons are an appealing choice for qubits.

Quasiparticles that obey non-Abelian statistics were first observed in materials exhibiting the fractional quantum Hall effect, which occurs in 2D electron gases exposed to strong magnetic fields. Unfortunately, these materials are impractical for generic quantum computation [3]. Another system that could host non-Abelian quasiparticles, such as anyons, is a 2D unconventional $p$-wave superconductor [4]. These superconductors are distinguished from conventional s-wave superconductors in the way that the spins are aligned in the Cooper pairs that carry the current. Physicists expect that the constituents of an anyon-known as Majorana zero modes-should appear in $p$-wave superconductors in the center of vortices that can form in the material. But these superconductors have proven hard to realize.

In theory, an s-wave superconductor can be converted to a $p$-wave superconductor by coupling a conventional s-wave 
superconductor with a semiconducting nanowire that has a significant spin-orbit interaction, in the presence of a moderate magnetic field. Some experimental evidence suggests that doing so leads to Majorana zero modes at both ends of the nanowire [5]. Equivalently, coupling the $s$-wave superconductor to a spatially rotating magnetic field could create a $p$-wave superconductor and Majorana zero modes without the need for the strong spin-orbit interaction [6].

Building on the concept of a rotating magnetic field, an intriguing idea for creating a Majorana-zero-mode hosting platform is to couple a vortex in a conventional superconductor to a magnetic vortex called a skyrmion [7]. In this magnetic structure (Fig. 1), the spins have a tilt that depends on their distance from the skyrmion center, and the "winding number" that describes the magnetic topology [8]. Most materials exhibiting skyrmions contain magnetic layers of iron $(\mathrm{Fe})$ or cobalt (Co) stacked with layers of heavy transition metals, such as platinum (Pt) or iridium (Ir), which provide the strong spin-orbit coupling necessary to form this magnetic structure.

Many theoretical works discuss the possibility of creating the desired skyrmion-vortex coupling in heterostructures that combine magnetic and superconducting layers. But experimental studies of these systems are still rare. Both parts of the heterostructure must stay within specific ranges of temperature and magnetic-field strength to realize the desired topological phase, and the length scales of skyrmions and vortices must be similar in order to study their coupling. In recent years, progress has been made in controlling the temperature and radius of skyrmions with moderate magnetic fields [9].

Now, Petrović and his colleagues have developed a heterostructure that achieves stable skyrmion-vortex coexistence, with control over the coupling between these two topological excitations. The researchers experimentally study the interactions between a magnetic [ $\mathrm{Ir} / \mathrm{FeCo} / \mathrm{Pt}]$ sandwich structure that can host skyrmions and a superconducting niobium $(\mathrm{Nb})$ layer that is either in direct contact with the magnet or separated by a thin insulating MgO layer [1]. A small applied magnetic field nucleates in the sandwich-structure skyrmions that have spins that rotate in the radial plane from the core to the periphery. Stray magnetic fields from the skyrmions induce vortices in the $\mathrm{Nb}$ film, which were observed with scanning tunneling spectroscopy.

The researchers explain the mechanism for the skyrmion-vortex induction in terms of an effect observed when a two-dimensional electron gas at an interface becomes spin polarized. This spin polarization leads to spin accumulation and, because of spin-orbit coupling, a charge current at the interface. Whether the spin polarization is induced by the exchange coupling in direct contact between the magnetic and superconducting layers or just by the stray magnetic field, it can be regulated by the presence or absence of the intervening insulator layer. Since temperature alters the ratio of the superconductor vortex radius to the skyrmion radius (each around $50 \mathrm{~nm}$ ), the coupling strength can be tuned.

Magnet-superconductor heterostructures offer a fascinating playground for creating topological phases that host exotic quasiparticles in a thin-film setup, and this demonstration of skyrmion-vortex coupling is an important step toward generating Majorana zero modes. However, it is still challenging to separate them spatially and energetically from other states so that they can be directly observed. The next step to doing that will be to create skyrmions of different shapes [7] or with magnetic ordering described by different winding numbers [10]. This step will require fine-tuning of the magnetic interactions and careful selection of the magnetic materials but, when interfaced with conventional superconductors, doing so should lead to increased stability of the Majorana zero modes. We can expect more exciting discoveries along the way to topological quantum computing.

Gustav Bihlmayer: Peter Grünberg Institute, Forschungszentrum Jülich, Jülich, Germany

\section{REFERENCES}

1. A. P. Petrović et al., "Skyrmion-(anti)vortex coupling in a chiral magnet-superconductor heterostructure," Phys. Rev. Lett. 126, 117205 (2021).

2. A. Yu. Kitaev, "Fault-tolerant quantum computation by anyons," Ann. Phys. 303, 2 (2003).

3. C. Nayak et al., "Non-Abelian anyons and topological quantum computation,” Rev. Mod. Phys. 80, 1083 (2008).

4. J. Alicea, "New directions in the pursuit of Majorana fermions in solid state systems," Rep. Prog. Phys. 75, 076501 (2012).

5. V. Mourik et al., "Signatures of Majorana fermions in hybrid 
superconductor-semiconductor nanowire devices," Science 336, 1003 (2012).

6. T. -P. Choy et al., "Majorana fermions emerging from magnetic nanoparticles on a superconductor without spin-orbit coupling," Phys. Rev. B 84, 195442 (2011).

7. S. Rex et al., "Majorana bound states in magnetic skyrmions imposed onto a superconductor," Phys. Rev. B 100, 064504 (2019).
8. S. Heinze et al., "Spontaneous atomic-scale magnetic skyrmion lattice in two dimensions," Nat. Phys. 7, 713 (2011).

9. A. Soumyanarayanan et al., "Tunable room-temperature magnetic skyrmions in Ir/Fe/Co/Pt multilayers," Nat. Mater. 16, 898 (2017).

10. G. Yang et al., "Majorana bound states in magnetic skyrmions," Phys. Rev. B 93, 224505 (2016). 